\title{
From Yeast to Patient Neurons and Back Again: Powerful New Discovery Platforms
}

\author{
Daniel F. Tardiff, PhD, ${ }^{1}$ Vikram Khurana, MD, PhD, ${ }^{1,2}$ Chee Yeun Chung, $\mathrm{PhD},{ }^{1}$ and Susan Lindquist, $\mathrm{PhD}^{1,3 *}$ \\ ${ }^{1}$ Whitehead Institute for Biomedical Research (WIBR), Cambridge, Massachusetts, USA \\ ${ }^{2}$ Department of Neurology, Massachusetts General Hospital and Harvard Medical School, Boston, Massachusetts, USA \\ ${ }^{3}$ Howard Hughes Medical Institute (HHMI), Department of Biology, MIT, Cambridge, Massachusetts, USA
}

\begin{abstract}
No disease-modifying therapies are available for synucleinopathies, including Parkinson's disease (PD), dementia with Lewy bodies (DLB), and multiple systems atrophy (MSA). The lack of therapies has been impeded by a paucity of validated drug targets and problematic cell-based model systems. New approaches are therefore needed to identify genes and compounds that directly target the underlying cellular pathologies elicited by the pathological protein, $\alpha$-synuclein ( $\alpha-s y n)$. This small, lipid-binding protein impinges on evolutionarily conserved processes such as vesicle trafficking and mitochondrial function. For decades, the genetically tractable, single-cell eukaryote, budding yeast, has been used to study nearly all aspects of cell biology. More recently, yeast has revealed key insights into the underlying cellular pathologies caused by $\alpha-$ syn. The robust cellular toxicity caused by $\alpha$-syn expression facilitates unbiased high-throughput small-molecule screening. Critically, one must validate the discoveries made in yeast in disease-relevant neuronal models. Here, we
\end{abstract}

describe two recent reports that together establish yeast-to-human discovery platforms for synucleinopathies. In this exemplar, genes and small molecules identified in yeast were validated in patient-derived neurons that present the same cellular phenotypes initially discovered in yeast. On validation, we returned to yeast, where unparalleled genetic approaches facilitated the elucidation of a small molecule's mode of action. This approach enabled the identification and neuronal validation of a previously unknown "druggable" node that interfaces with the underlying, precipitating pathologies caused by $\alpha$-syn. Such platforms can provide sorely needed leads and fresh ideas for disease-modifying therapy for these devastating diseases. (C) 2014 International Parkinson and Movement Disorder Society

Key Words: $\alpha$-synuclein; Parkinson's disease; phenotypic screen; yeast; human iPS cells; drug target identification
${ }^{*}$ Correspondence to: Dr. Susan Lindquist, 9 Cambridge Center, Cambridge, MA 02142, E-mail: lindquist_admin@wi.mit.edu

Funding agencies: S.L. is an investigator for HHMI. D.F.T. was funded by a National Research Service Award (NRSA) fellowship F32NS061419, the Thome Memorial Foundation, and research supported by the JPB Foundation (D.F.T. and S.L.), the Eleanor Schwartz Charitable Foundation, and an HHMl Collaborative Innovation Award (D.F.T., V.K., C.Y.C., S.L.). V.K. was funded by an American Brain Foundation and Parkinson's Disease Foundation Clinician-Scientist Development Award, and the Harvard Neurodiscovery Center Pilot Project Program. C.Y.C. was supported by funding from the NIH/NIA (K01 AG038546).

Relevant conflicts of interest/financial disclosures: Nothing to report. Author roles may be found in the online version of this article.

Received: 2 June 2014; Revised: 10 July 2014; Accepted: 14 July 2014

Published online 14 August 2014 in Wiley Online Library (wileyonlinelibrary.com). DOI: 10.1002/mds.25989
The continued aging of our population and our current inability to treat neurodegenerative diseases are poised to precipitate a societal and economic crisis. Despite large investments in Alzheimer's disease and Parkinson's disease (PD) research, results have fallen far short of expectations. Unclear disease etiologies, unproven biomarkers, slow disease progression, and challenges in stratifying patients in clinical trials are compounded by the challenges in early-stage research, where the validity of in vitro systems and model organisms are under constant scrutiny. Taken together, identifying disease-modifying therapies is a tremendous challenge. However, rapidly improving methodologies and an evolving mechanistic understanding of disease is fostering more interdisciplinary approaches to research. Delivering disease-modifying 
TARDIFF ET AL)

therapies to patients will require collaborative endeavors that exploit diverse expertise and innovative approaches.

Two recent papers from our group and several collaborators in Science $e^{1,2}$ provide an example of this multi-faceted approach. We built on prior work to establish yeast-to-human discovery and validation platforms that provides a significant proof-of-principle: lessons learned in yeast illuminate our understanding of human neuronal pathologies and can fuel our efforts to correct them. The goal of this invited Scientific Perspective is to make this work, which was originally presented in a condensed, difficult-to-digest format, more accessible to a broader audience and to provide context for the potential of our platform for drug discovery and development. We hope that this platform will inject new ideas and tools for pursuing therapeutic strategies against PD and other neurodegenerative diseases.

\section{Some Background: Yeast as a Model System for Synucleinopathies}

First and foremost, we make no claims about modeling human disease in yeast. Rather, our laboratory and that of many others model the early, precipitating cellular pathologies caused by $\alpha-$ synuclein $(\alpha-$ syn) in a simple eukaryotic cell. ${ }^{3}$ This small lipid-binding protein contributes to a class of synucleinopathies, including PD, dementia with Lewy Bodies (DLB), and Multiple Systems Atrophy (MSA). Despite significant evolutionary distance, basic cell biological processes are highly conserved from yeast to humans. Vesicle trafficking pathways, mechanisms that maintain proper protein folding, mitochondria, and lipid biology, for example, are fundamentally similar from yeast to humans.

As a single-cell 'living test tube,' yeast cells do not make the extensive cellular connections that neurons do through synapses. Paradoxically, this feature of yeast provides some key advantages. When focusing on initiating events in protein pathologies, experiments are not confounded by complex interactions with other cell types, such as astrocytes and glia. Initially, the single cellularity enables one to perform genetic and biochemical analyses to investigate the initial pathologies in temporally controlled fashion in genetically and epigenetically identical cells. Notably, however, the phenotypes we observe, even in yeast, are not strictly cell autonomous.

As in human neurons, $\alpha$-syn binds lipids in yeast and travels on vesicles to the cell periphery. When $\alpha$ syn levels increase only about twofold, there is a dramatic change in localization to intracellular foci composed of $\alpha$-syn-decorated trafficking vesicles. This change in localization is accompanied by cellular toxicity ('toxicity' is defined as a reduction in cell growth followed by cell death). The sharp dose-dependent increase in toxicity of wild-type $\alpha$-syn also occurs in humans, in whom duplication or triplication of the wild-type $\alpha$-syn gene causes early-onset PD. ${ }^{5-7}$ Furthermore, single-nucleotide polymorphisms in the 3, UTR increase $\alpha$-syn levels and confer disease risk. ${ }^{8,9}$ Notably, the $\alpha-$ syn foci in yeast are not Lewy bodies (LB), but rather they reflect early pathological effects of $\alpha$-syn on vesicle trafficking. Likely a much longer period of time, such as that in a PD patient, is required to get bona fide LBs.

The cellular toxicity caused by $\alpha-\operatorname{syn}$ provided a simple readout in an unbiased screen for genes that make cells grow better (suppress toxicity) or worse (enhance toxicity). The function of the identified genes would provide information as to how $\alpha$-syn impinges on normal cell physiology. After testing nearly all 6,000 yeast genes, only approximately 60 suppressed $\alpha$-syn toxicity. ${ }^{10,11}$ The largest class of genetic hits promoted the trafficking of vesicles from the endoplasmic reticulum (ER) to the Golgi. The value of such unbiased, genome-wide approaches is that they proved signposts to the fundamental nature of the pathology. Here, the fact that genes promoting vesicle trafficking rescued $\alpha$-syn toxicity suggested that $\alpha$-syn inhibits vesicle trafficking. ${ }^{10}$

Indeed, biochemical analyses showed that $\alpha$-syn caused a dramatic inhibition of ER-to-Golgi vesicle trafficking. ${ }^{10,12}$ Further work has indicated that many other aspects of vesicle trafficking, such as endosomal transport, or the internalization of membrane proteins from the cell surface, are also affected by $\alpha$-syn. ${ }^{4,12-16}$ Importantly, the phenotypes identified in our yeast screens have been validated in several mammalian and neuronal models of $\alpha$-syn toxicity. ${ }^{10,17-22}$ Moreover, several human PD risk factors (see below) also function within the trafficking pathways identified in unbiased yeast screens, ${ }^{23-30}$ suggesting that early toxic events revealed in yeast have direct relevance to precipitating pathologies in disease.

In further validation, the yeast screen directly linked a known PD risk allele-PARK9-to $\alpha$-syn toxicity. ${ }^{31}$ Mutations in PARK9 (ATP13A2) cause an early-onset Parkinsonism previously not connected to $\alpha$-syn. In yeast, overexpressing the PARK9 homolog, Ypk9, suppresses $\alpha$-syn toxicity through modulating metal homeostasis. This interaction holds true in mammalian cells and neurons where PARK9 influences vesicle trafficking and metal homeostasis. ${ }^{32-35}$ Thus, the unbiased $\alpha$-syn yeast screen was able to establish connections where they were previously unknown.

While there is substantial support for the utility of the yeast model, we do not take lightly potential limitations. One frequent question that arises is how a 
single-celled organism such as yeast might give clues to the differential vulnerability of specific groups of neurons and glial cells to neurodegenerative diseases. We suggest that the panel of specific cellular responses and pathologies in response to misfolded proteins in yeast can illuminate why specific cell types might be vulnerable or resistant to degeneration. Nevertheless, there will undoubtedly be neuronal-specific mechanisms, phenotypes that require interactions between different cell types, or particular neuronal activities that cannot be modeled in this unicellular organism. In addition, the potential impact of $\alpha$-syn spreading mechanisms may not be fully realized. Interestingly, however, prion-like templating of protein conformers from mother to daughter cells in yeasts has been extensively studied. None of these "limitations" precludes yeast from faithfully recapitulating the earliest cellular pathologies. Even if some relationships fail to validate in neuronal models, the unparalleled discovery capabilities of this model outweigh any potential drawbacks. Moreover, human genetic studies rationalize our approach because, far from being neuronspecific, many genes tied to PD and other neurodegenerative disorders are ubiquitously expressed genes highly conserved across evolution.

\section{Rationale Behind Yeast-to-Human $\alpha$-syn Platforms}

The two original questions driving the two research papers in question were conceived independently. In Tardiff et al., ${ }^{1}$ we asked two questions: Can we identify small molecules from unbiased chemical screens that correct multiple toxic phenotypes associated with $\alpha$-syn? And, if so, might we take advantage of yeast genetics to identify that compound's protein target and mechanism of action? The concept was to use the cells themselves to identify a "druggable" node to rescue cells from $\alpha$-syn toxicity, without introducing our own prejudices about what might be a "good druggable target." In Chung, Khurana, et al., ${ }^{2}$ we asked whether yeast biology could help us break through a roadblock in the use of induced pluripotent stem (iPS) cells to study diseases of advanced age. Could yeast phenotypes point to early cellular pathologies for synucleinopathies in patient neurons, without the need to resort to the use of toxins such as $\mathrm{H}_{2} \mathrm{O}_{2}{ }^{36-38}$ ? Bridging these two manuscripts was the observation that the compound identified and characterized in Tardiff et al. rescued the phenotypes caused by $\alpha$-syn mutations in PD patient neurons, and did so via the same mechanism. Together, these two papers present an array of complementary methodologies and model systems that describe the potential for yeast-to-human neuron platforms for the identification and validation of drugs and drug targets.

\section{Yeast Reveal a "Druggable" Rsp5/ Nedd4 Network That Ameliorates Alpha-Synuclein Toxicity in Neurons (Tardiff et al.)}

\section{Investigation Rationale}

A limitation in identifying disease-modifying therapies for neurodegenerative diseases is a lack of validated targets that both can be targeted with small molecules and are key nodes central to the underlying, causative cellular pathologies. To date, drug discovery has been intently focused on target-based approaches. Functionally validated targets are adapted to in vitro assays and screened against hundreds of thousands of compounds to identify those that modulate this target. While having the distinct advantage of being able to identify high-potency compounds with a known target, these assays are far removed from the extremely complex cellular milieu within which these targets actually function. Be it intracellular localization, interacting partners, or posttranslational modifications, numerous complicating factors may mitigate the efficacy of a given compound in vivo. Moreover, offtarget effects, an inability to enter cells, or metabolism can thwart in vivo efficacy. Finally, and perhaps most importantly, the ability of biological systems to adapt to the targeting of particular proteins is impossible to predict.

An alternative pathway to drug discovery is to screen in cells for compounds that reverse a robust phenotype, such as cellular toxicity. ${ }^{39}$ Such phenotypic screens are completely unbiased, having no preconception of what makes for a good target. This unbiased, cell-based approach surveys hundreds of thousands of compounds in a living cell with its full complement of cellular factors and complexities that are missing in target-based screens. In fact, although such screens represent a minority of total efforts in the pharmaceutical industry, this approach has had a higher success rate in producing FDA-approved drugs than target-based screens. ${ }^{40}$ The single biggest barrier in adapting phenotypic smallmolecule screens to drug discovery is the difficulty of identifying the molecules mechanism of action (MOA). Such knowledge greatly empowers clinical trial procedures. Here yeast cells offer a remarkable advantage; the organism provides an unparalleled tool box, fully stocked with diverse but complementary genetic approaches for target identification. ${ }^{41}$

The ability of yeast to identify compounds that target conserved proteins is exemplified by the ability of several blockbuster drugs-including the statins, omeprazole, tacrolimus (FK-506), bortezomib (Velcade), and methotrexate- to hit the same highly conserved targets and elicit the same responses in yeast as they do in humans. Although this established conservation 
TAR IFF ET AL)

does not guarantee all compounds will cross over into mammalian systems, the phenotypic screens in yeast serve as a robust first filter for compounds with cytoprotective activity.

\section{Manuscript Synopsis}

We started with a compound that rescued yeast cells from $\alpha$-syn toxicity but did not rescue them from amyloid- $\beta(A \beta)$, polyglutamine, and fused in sarcoma/ translocated in liposarcoma (FUS/TLS) toxicity. In addition to restoring growth, this $\mathrm{N}$-arylbenzimidazole (NAB) reversed key $\alpha$-synphenotypes in yeast: it restored localization of $\alpha$-syn to the plasma membrane, it rescued the ER-Golgi trafficking defect, and it prevented generation of reactive oxygen species. Critically, $\mathrm{NAB}$ also partially reversed toxicity caused by $\alpha$-syn overexpression in both nematode (C. elegans) neurons and rat primary midbrain neuronal cultures. Thus, it seemed highly likely that (1) NAB's target was conserved from yeast to mammals and (2) the target mattered for $\alpha$-syn pathology.

We first noticed that just above its maximal effective dose, NAB caused cells to grow more slowly, creating a bell-shaped dose-response curve. At these concentrations, NAB also slowed growth of wild-type yeast cells. Was reduced growth caused by an off-target interaction, or was it caused by excessive modulation of the same target that was responsible for rescue? To address this, and to get a better understanding of the critical structural features of the compound, our organic chemistry collaborators synthesized analogs that tested the importance of NAB's various structural features. A key observation was made: Compounds that were more potent against $\alpha$-syn toxicity were also more potent in inhibiting the growth of wild-type cells when used at modestly higher concentrations. The strong correlation between efficacy and growth inhibition suggested that growth inhibition was not attributable to a spurious off-target effect. Notably, NAB did not reduce viability at any concentration; it was cytostatic. This enabled us to employ multiple chemical genetic screens for target identification.

Because NAB2 inhibited the growth at higher concentrations, we used this simple phenotype to screen for genes that-when overexpressed or mutated-conferred resistance to NAB2. We did so in cells not expressing human $\alpha$-syn. Identifying such genes in wild-type yeast would provide insights into targeted pathways, and potentially the direct target for NAB2. We took advantage of diverse types of genome-wide screens that can currently only be done easily in yeast (Fig. 1A).

First, we screened an overexpression library, a collection of 6,000 yeast strains each overexpressing a single gene when induced by a specific signal. Cells that make higher levels of the target protein, or of other critical proteins in the target's pathway, should be resistant to high NAB2 concentrations. Second, we used a library of approximately 300,000 strains wherein each cell carried a single transposon randomly inserted into the yeast genome. These can disrupt open reading frames or regulatory elements to modulate expression. By selecting for rare variants that could grow in high concentrations of NAB, we garnered insights into genes that might be the target, or closely related to the target. Finally, we selected strains that had spontaneous mutations that conferred resistance to NAB2. These could be identified by sequencing the entire genome of these cells.

From the combination of these stringent screens, we obtained 12 genes that conferred resistance to NAB2 when mutated or overexpressed. We next took advantage of the vast collection of genetic and proteomic data sets available in yeast to identify connections among the screen hits. From this analysis, it was clear that the genes formed a highly connected network of proteins that function in ubiquitin-dependent endocytosis and the lysosomal targeting of membrane proteins. The center of this network was the homologous to the E6-AP carboxyl terminus (HECT) domain E3 ubiquitin ligase, Rsp5 (Nedd4 in humans). This ligase ubiquitinates membrane proteins and directs their endosomal transport, rather than targeting them for degradation by the proteasome. ${ }^{42}$

Which member in this collection of genes was the actual target? Further work established that 11 of the 12 cannot be the target. We deleted each one, and although some altered the efficacy of NAB2, none eliminated it. In yeast, approximately $80 \%$ of genes are not essential for viability. Rsp5, the lone essential gene and center of the network, could not be deleted and was thus most likely to be target. The nature of the genetic interactions within the network indicated that we were activating Rsp5 with NAB2, not inhibiting it. For example, mutations that compromised Rsp5's activity in endosomal transport conferred resistance to NAB2. This indicated that NAB2 had the opposite effect: it promoted Rsp5-dependent trafficking. The suggestion that NAB2-activated Rsp5-dependent trafficking was confirmed in functional cell-based assays. NAB2 promoted endosomal trafficking of three independent proteins in a manner that depended on functional Rsp5 (Fig. 1B).

Because these studies on NAB2's MOA were performed in cells not expressing $\alpha$-syn, we next tested whether the Rsp5 network was directly relevant to NAB's rescue of $\alpha$-syn toxicity. Indeed, deleting genes involved in endocytosis, mutating Rsp5 itself, or overexpressing deubiquitinases all mitigated the ability of NAB2 to rescue $\alpha$-syn toxicity (Fig. 1C). This indicated that the chemical genetic network we identified in wild-type cells was directly related to NAB2's ability to ameliorate $\alpha$-syn toxicity. In fact, a single 


\section{A. Chemical Genetic Discovery of Target Space}
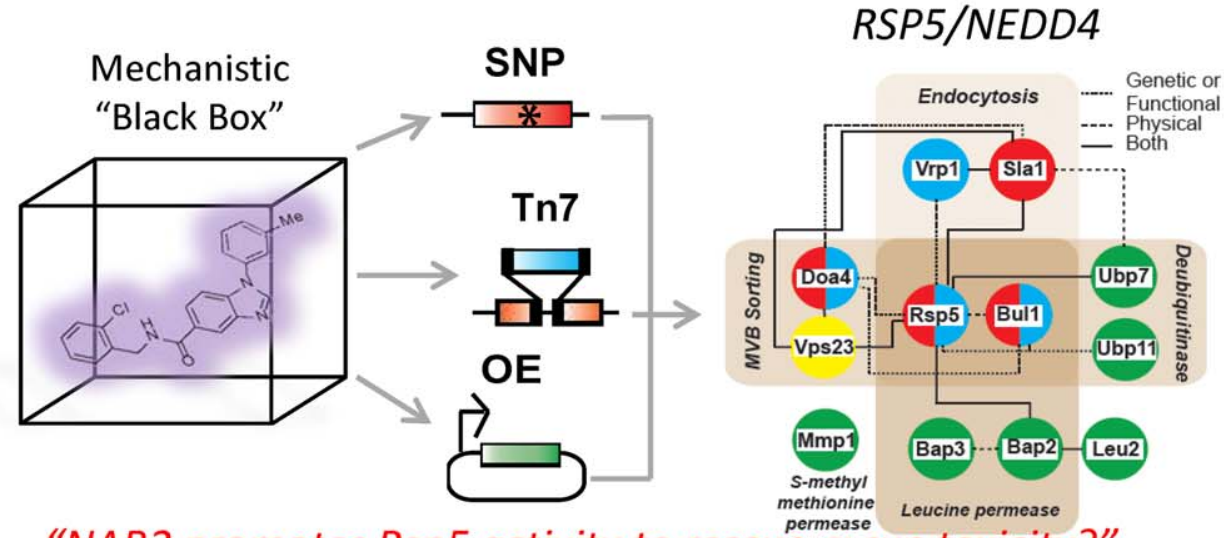

"NAB2 promotes Rsp5 activity to rescue $\alpha$-syn toxicity?"

\section{B. NAB2 promotes Rsp5-dependent endosomal transport}

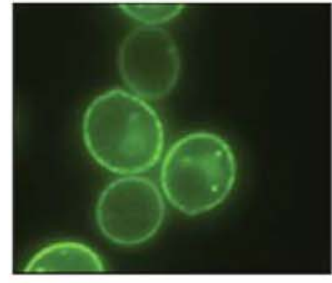

Normal Condition

WT cells with MUP1-GFP (no $\alpha$ Syn)

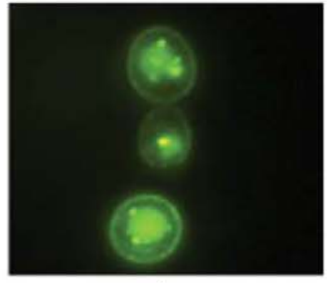

+ NAB2 / WT Rsp5

(promotes endocytosis)

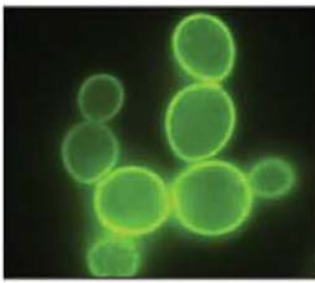

+ NAB2 / Rsp5 mut

(does not promote endocytosis)

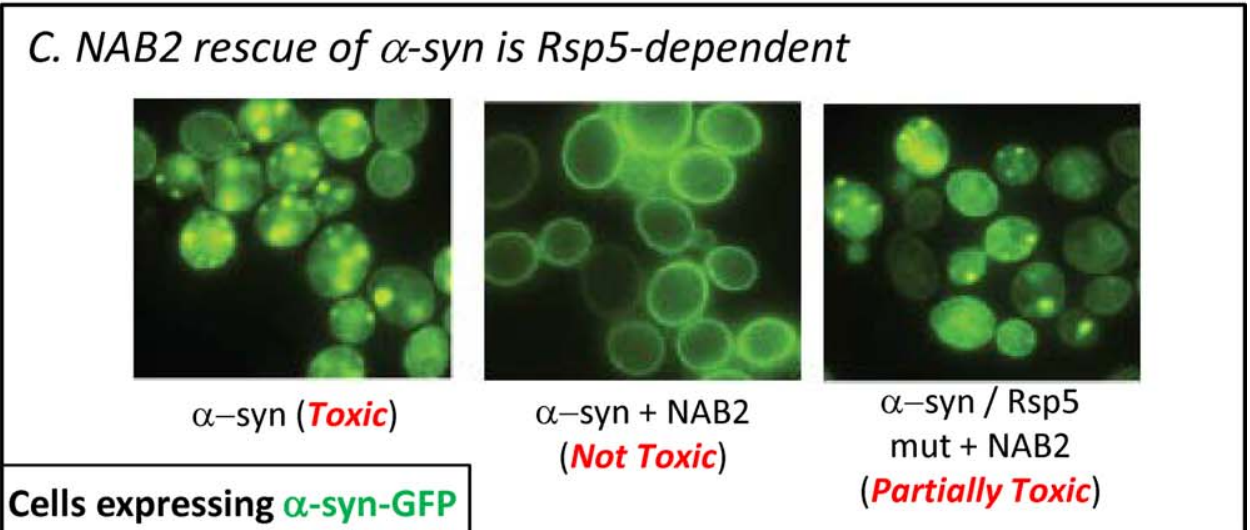

FIG. 1. Yeast chemical genetics identifies conserved mode of action for NAB. (A) Because the MOA for NAB was completely unknown, we used three independent genetic screens in yeast to identify a network of genes that modulated NAB2 activity. Together, the identity of the genes and the directionality of modulation suggested that NAB promotes Rsp5-dependent endosomal trafficking. (B) Using functional cell-based assays (Mup1-GFP, a known Rsp5 substrate, shown here), NAB2 was shown to promote the endosomal transport of multiple proteins in a manner that was dependent on wild-type Rsp5 activity. (C) Finally, the relevance of the Rsp5 network for NAB's rescue of $\boldsymbol{\alpha}$-syn toxicity by examining rescue and toxic phenotypes. The same mutations that confer resistance to NAB2 in wild-type cells lacking $\boldsymbol{\alpha}$-syn, also mitigated rescue of $\boldsymbol{\alpha}$-syn toxicity and foci reversal.

amino-acid substitution in the approximately 800 amino-acid Rsp5 protein mitigated NAB2's reversal of $\alpha$-syn phenotypes to inhibit the growth of otherwise wild-type cells.

Thus, Rsp5 and endosomal transport seemed deeply rooted in the underlying cellular toxicity caused by $\alpha$ - syn. To test this directly, we examined the same cellular reporters of Rsp5 activity used in our MOA studies. Indeed, $\alpha$-syn impaired the endosomal transport of these proteins. By exploiting the extensive genetic and interactome databases, it also became apparent that the $\alpha$-syn genetic network was highly interconnected 
TARDIFF ET AL)

with the NAB2 network. Thus, $\alpha$-syn inhibits several vesicle trafficking steps in one direction, whereas NAB2 and Rsp5 promotes some of these same steps in the other direction.

There are approximately 600 ubiquitin ligases in the human genome, and they profoundly affect protein homeostasis in a myriad of ways. Yet, they have been essentially "undruggable," let alone subject to chemical activation, an even rarer mode of small-molecule action. Also, absolutely pivotal to our goals, Rsp5 is highly conserved across evolution, including several mammalian homologs, as part of the NEDD4 family, that have similar functions. ${ }^{42}$ These ligases function in a highly complex manner, whereby different domains within the full-length protein bind to calcium, lipids, adaptor proteins, substrates, and deubiquitinases to modulate activity, as well as be subject to autoinhibitory conformations and post-translational modifications. It is therefore conceivable to regulate a Nedd4type ubiquitin ligase through several mechanisms.

This paper provides an example and path forward to identify the MOAs of compounds identified from phenotypic small-molecule screens of neurodegenerative disease-relevant cellular toxicities. Few fully unbiased phenotypic small molecule screens have been performed against cell-based $\alpha$-syn toxicity models, ${ }^{39}$ largely because of the difficulty in establishing robust cellular models amenable to the required high throughput screening formats. Yeast models accomplished this task and have the distinct advantage of diverse genetic approaches to identify small molecule targets and pathways. Also, showing that these genes were relevant to rescuing $\alpha$-syn toxicity was critical to validating the relevance of the NAB2 network. A priori, it was not clear that Rsp5 was a node central to the pathologies caused by $\alpha$-syn, let alone one that could be targeted with small molecules. Of course, it will be essential to test NAB2 and Nedd4 in animal synucleinopathy models with their highly interconnected neuronal networks. Both for NAB2 and future compounds, animal testing may first require compound optimization for both efficacy and in vivo pharmacology.

\section{Identification and Rescue of Alpha- Synuclein Toxicity in Parkinson Patient-Derived Neurons (Chung, Khurana et al)}

\section{Investigation Rationale}

Although many forms of parkinsonism exist, some without and some with synuclein pathology, Parkinson's disease by definition is parkinsonism associated with synuclein pathology involving the pars compact of the substantia nigra. Most parkinsonism is sporadic
Parkinson's disease with synuclein pathology. Mutations and gene duplication/triplication of the $\alpha$-syn gene can cause familial forms of PD, and singlenucleotide polymorphisms in synuclein confer risk for late-onset sporadic PD. These findings causally link $\alpha$ syn to the disease process, and makes $\alpha$-syn accumulation the best-defined molecular determinant for PD and related disorders. Having relevant cellbased synucleinopathy models is essential to understand disease pathogenesis and identify/validate potential drug targets. Recent advances in stem cell technologies, namely, the ability to reprogram mature somatic cells of patients into pluripotent "induced pluripotent stem (iPS) cells" that can be differentiated into disease-relevant cell types, ${ }^{43}$ may provide a gamechanging avenue to exploring disease mechanism, patient stratification, and perhaps eventually personalized medicine.

A critical issue with induced pluripotent stem (iPS) cell modeling of disease pathologies is having the appropriate control lines. Because every person is genetically different, innumerable genetic differences may influence a cellular phenotype observed from cells grown in a dish. ${ }^{44}$ Comparing a single mutationcorrected line with a parental line is far more desirable than comparing numerous wild-type controls with a similar number of unrelated patient samples.

Another major hurdle is identifying cellular pathologies, or phenotypes, that are directly caused by the predisposing disease mutation. To date, most analyses of iPSc-based systems rely on an exogenous toxin to exacerbate an underlying condition. We hoped to uncover innate phenotypes that are independent of secondary insults. To begin our exploration of these patient cell lines, we relied on our simple yeast $\alpha$-syn toxicity model to guide our investigation of phenotypes (Fig. 2).

\section{Manuscript Synopsis}

We began our investigation of $\alpha$-syn toxicity using cells derived from known kindreds of patients with the A53T $\alpha$-syn point mutation and a genetically identical control in which the mutation was corrected using genome-editing technologies. ${ }^{45}$ Additionally, we analyzed cells reprogrammed from a patient who had a triplication of the wild-type $\alpha$-syn gene. The goal was then to identify phenotypes in a disease-relevant class of neurons. Although PD is often associated with the loss of dopaminergic neurons in the substantia nigra, profound cortical pathology also occurs later in the disease. Interestingly, DLB, another synucleinopathy, presents with severe cortical pathology that precedes dopaminergic neuron degeneration. Because dementia and cortical pathology are particularly prominent features in patients harboring mutations in the $\alpha$-syn gene and because large numbers of cortical 


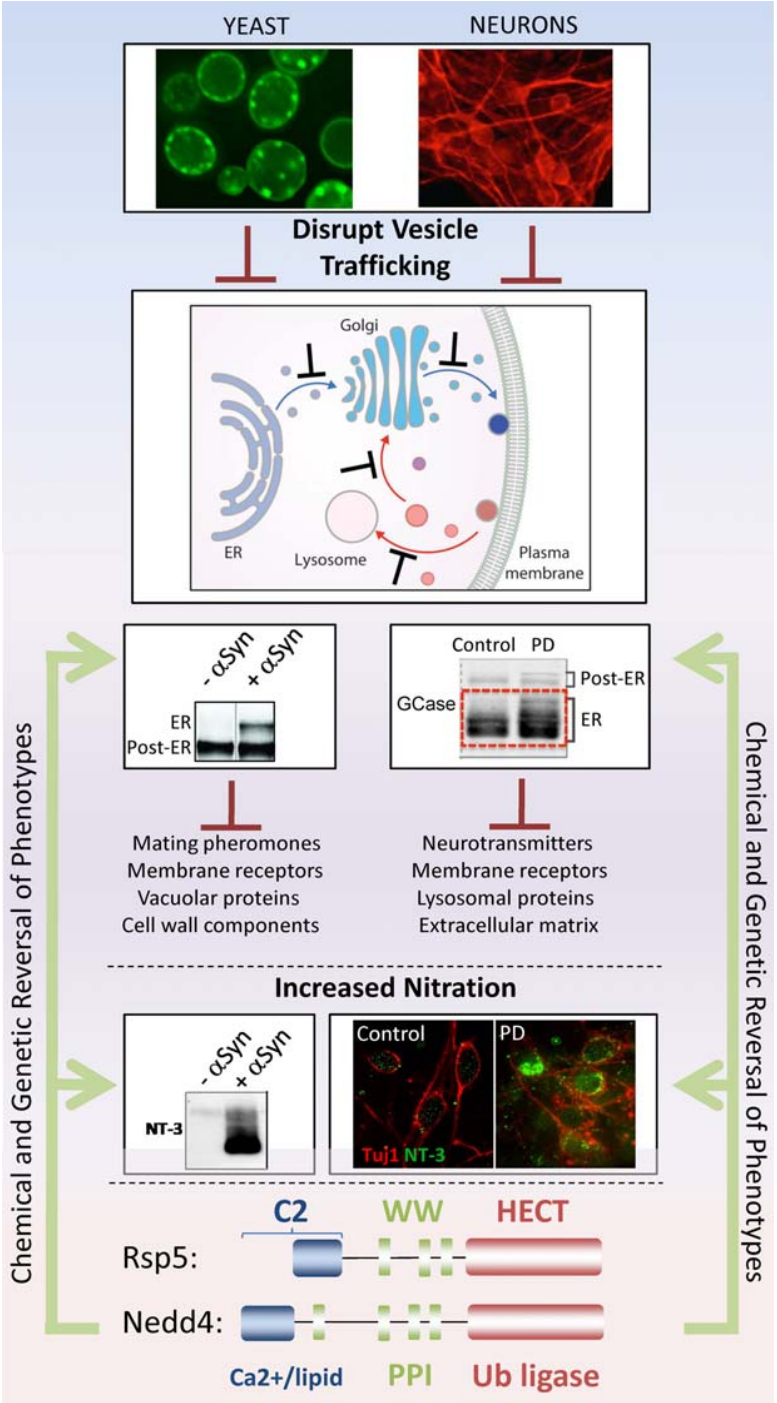

FIG. 2. Schematic of yeast-to-human platforms. The discovery/ validation platforms exploit the fact that the fundamental cellular processes and proteins are highly conserved from simple yeast cells all the way to humans, including complex neurons. Using yeast for robust discovery and patient neurons for disease-relevant validation, genes and compounds that ameliorate toxicity and phenotypes in yeast similarly reverse these phenotypes in patient-derived neurons. In both cell types, $\boldsymbol{\alpha}$-syn both negatively impacts aspects of vesicle trafficking and protein quality control and causes an increase in protein nitration. These phenotypes were directly revealed by unbiased genetic screens in yeast. A final critical aspect entails a return to yeast genetics to elucidate the MOA of new compounds and reveal unanticipated "druggable" nodes that can fix the underlying cellular dysfunctions caused by $\alpha$-syn in both yeast and human neurons. This approach of integrating multiple genetic screens identified the MOA for NAB2, an activator of the HECT domain E3 ubiquitin ligase, $R S P 5 / N E D D 4$.

neurons can readily be generated from iPS cells, we chose to differentiate patient-derived iPS cells into cortical neurons for our study. After choosing the relevant cell type, the next hurdle was to establish innate phenotypes that were specific to $\alpha$-syn pathology, rather than resorting to exogenous toxins that may report on a pathology distinct from the precipitating molecular consequences of $\alpha$-syn perturbation.
We first heeded the lessons from the simple yeast model of $\alpha$-syn toxicity and focused on two suppressors of $\alpha$-syn toxicity: a nitrosative stress transcription factor and a facilitator of ER-associated protein degradation (ERAD). Two questions were posed: (1) Are the processes affected by these genetic modifiers similarly perturbed in both yeast and patient-derived neurons; and (2) Can increasing the levels of these proteins reverse the said phenotypes in a model or patient-derived neurons?

The identification of Fzf1, a transcription factor that responds to nitrosative stress, as a suppressor of $\alpha$-syn toxicity led us to evaluate protein nitration in response to $\alpha$-syn. In yeast, $\alpha$-syn caused a dramatic and specific increase in nitrotyrosine-modified proteins that was not detected in other proteinopathy models (i.e., TDP-43, $A \beta$, and so forth). In our iPSc model, we used a dye that monitors NO levels to evaluate whether a similar increase in nitrosative stress occurred in response to $\alpha$-syn mutation. Indeed, we detected a significant increase in NO levels, both in rat cortical neurons expressing $\alpha$-syn and in our human A53T and $\alpha$-syn triplication PD lines. This increase in $\mathrm{NO}$ was accompanied by an increase in nitrotyrosine itself in the patient-derived neurons.

We validated our findings in postmortem brain samples generated from another patient with PD harboring the A53T mutation. Here, too, we detected increased protein nitration in postmortem cortex of an A53T-harboring patient. ${ }^{46}$ Thus, the nitrosative stress signal in response to $\alpha$-syn toxicity was conserved from yeast to human. Moreover, these data suggest that the ultimate increase in protein nitration observed in patient brain samples likely has roots in early, precipitating cellular pathologies.

The second key phenotype we investigated was the accumulation of proteins that are normally degraded by ERAD. In this process, misfolded proteins in the ER are targeted for degradation by the proteasome. Hrd1, the yeast E3 ligase that ubiquitinates ERAD substrates, suppresses $\alpha$-syn toxicity in yeast. This suggests that ERAD is directly compromised by $\alpha$-syn. We then monitored multiple ERAD substrates in patient-derived neurons using a sensitive biochemical assay that detects protein trafficking intermediates because of differential glycosylation. Both glucocerebrosidase (GCase), which itself is a risk factor for $\mathrm{PD},{ }^{47}$ and Nicastrin accumulated in the ER of A53T patient-derived neurons. Importantly, viral delivery of the human homolog of Hrd1, Synoviolin, reversed the ERAD phenotype. In A53T and sporadic PD postmortem brain, we also detected accumulation of GCase in the ER. Thus, $\alpha$-syn-dependent phenotypes identified in yeast were conserved in neurons in vitro and in PD patient brain.

The validation of these two phenotypes in diseaserelevant neurons indicates that the yeast model of 
TARDIFF ET AL)

$\alpha$-syn toxicity can successfully predict innate cellular phenotypes that are perturbed in patient-derived neurons. Critically, these phenotypes developed in the absence of any additional exogenous toxin within 8 to 12 weeks of differentiation. Whether this time period would be sufficient time to develop phenotypes in a disease process that could take decades to develop in a patient brain was not initially clear. However, without increasing $\alpha$-syn levels or adding a toxin, pathological phenotypes developed before overt toxicity. Manifestation of these early phenotypes suggests that they are perhaps precipitating cellular disturbances or vulnerabilities that lead to subsequent cell death.

In Tardiff et al., ${ }^{1}$ we found that NAB2 rescued yeast, nematode, and rat neurons from $\alpha$-syn toxicity. As a final question in Chung, Khurana et al., ${ }^{2}$ we therefore asked whether this compound also reversed pathological phenotypes in patient-derived neurons. First, we demonstrated that NAB2 reversed the pathological ERAD substrate accumulation and nitrosative stress phenotypes in yeast. Then, both the A53T and $\alpha$-syn triplication cell lines were treated with NAB2. ERAD substrate accumulation and nitric oxide levels phenotypes were in fact reversed by NAB2 in patient-derived neurons.

Finally, to close the yeast-human loop, we tested whether the putative target of NAB2 established in yeast-the ubiquitin ligase Nedd4/Rsp5-reversed the pathological phenotypes. Indeed, Nedd4 overexpression alone restored mature forms of both GCase and Nicastrin. The ability of Nedd4 overexpression to mimic the effect of NAB2 indicates that the activation of Nedd4 is an important protective activity in directly antagonizing $\alpha$-syn -mediated cellular pathologies. Most importantly, however, the finding validated yeast as a discovery platform to identify small molecule targets and mechanisms in disease-relevant patient neurons.

\section{Translational/Clinical Perspective}

Our work suggests that two areas of early-stage drug discovery, which desperately need improvement, might be addressed by iteratively moving between simple cellular models and human cells and tissues. Tardiff et al. ${ }^{1}$ focuses on the idea of a lack of diseaserelevant "druggable" targets, and new ways are needed to identify such nodes. Chung, Khurana, et al. ${ }^{2}$ formulate an approach that exploits the conserved underlying biology between yeast and neurons to guide the identification of phenotypes in patientderived neurons. Identifying phenotypes in patient lines with distinct predisposing mutations and pathologies may be essential to predicting therapeutic potential. Although the translational impact of these reports is at an early stage, their insights might help provide a complementary approach to drug discovery and early clinical development.

\section{"Druggable" Nodes in Synucleinopathies}

Significant gaps are present in our understanding of the cellular pathologies causing neurodegenerative disease. This presents an extreme challenge in determining productive "druggable" nodes that can ameliorate underlying cellular pathologies. Through unbiased drug and genetic screening in yeast, we have revealed critical nodes that can be pharmacologically modulated to suppress the underlying causes of $\alpha$-syn toxicity. Specifically, the modulation of endosomal transport through activating $\mathrm{Rsp} 5 / \mathrm{Nedd} 4$ reflects a key node that directly antagonizes the effects of $\alpha$-syn on endosomal transport in cellular models.

In isolation from the context of actual human disease, the significance of an association in a model organism is not always clear. Critically, however, a number of PD genetic risk factors function in related trafficking pathways. Alpha-synuclein regulates synaptic vesicle transport $^{48}$; VPS35 is a component of the retromer complex that functions in endosome to Golgi transport ${ }^{26,27}$; LRRK2 is implicated in trans-Golgi protein sorting and synaptic endocytosis ${ }^{24,28}$; RAB7L1 is a GTPase functioning in trafficking ${ }^{23}$; GAK is a cyclin-dependent kinase that regulates clathrin-coated vesicles and lysosomal transport ${ }^{25,30}$; and DNAJC13 functions in endosomal transport. ${ }^{29}$ Among these proteins, LRRK2 interacts physically with both RAB7L $1^{23}$ and GAK. ${ }^{49}$ Thus, the convergence of these pathways in causing PD suggests that trafficking defects play a critical role in disease progression. This is especially germane to neurons where synaptic vesicle release and uptake is critical to maintain neuronal function.

We have shown that NAB2 will be an important biological probe for studying $\alpha$-syn toxicity and other biological pathways that are dependent on Rsp5/Nedd4. However, is Nedd4 a good drug target for PD? The role of Rsp5/Nedd4 in promoting endosomal transport appears poised to antagonize a dysfunction that is emerging as a central cellular pathway implicated in PD. Although a direct protective role for Nedd4 against $\alpha$-syn toxicity has also been suggested by its ability to ubiquitinate $\alpha$-syn and promote degradation, ${ }^{50}$ we did not observe a significant decrease in $\alpha$-syn levels. Thus, NAB2 distinctly targets the underlying cellular pathologies elicited by $\alpha$-syn directly. Support for this also stems from how both NAB and its mode of action were revealed. First, NAB2 was originally identified based on its ability to rescue TDP-43 toxicity. Second, NAB2 mode of action was elucidated in the context of a wild-type cell without $\alpha$-syn being present. Regardless, Nedd4 is present within the neurons expressing $\alpha$ syn and even co-localizes to LB. ${ }^{50}$ Nedd4 therefore appears to be a central node within the $\alpha$-syn network, and is localized to a salient cell type, where its modulation could impact the progression of disease phenotypes. Clearly, Nedd4 will not be the only "druggable" 
target capable of ameliorating the underlying cellular pathologies elicited by $\alpha$-syn. NAB2 and Rsp $5 /$ Nedd 4 do, however, provide a possible blueprint for identifying new molecular entities and drug targets. Integrating unbiased drug screens with chemical genetic target identification will likely reveal new, unanticipated nodes that directly target $\alpha$-syn toxicity.

\section{Patient iPS Cells: From Dish to Clinic?}

The spectrum of genetic and sporadic causes of synucleinopathy etiologies suggests that not all patients experience the exact same underlying cellular pathologies. This inherent complexity creates a significant challenge when recruiting patients for clinical trials. Stratifying patients based on innate cellular phenotypes may ultimately maximize the potential for identifying successful therapies. Particularly relevant to the current discussion are the expanding capabilities to reprogram, genetically correct, and differentiate patient cells into disease-relevant neurons. This offers potential to identify phenotypes and, as an extension, patients, that might respond to a compound with a specific mode of action. By elucidating cellular pathologies in diverse patient-derived neurons, we may be able to assign compound-phenotype pairings to more accurately identify which patients may respond to a given therapy. Thus, identifying and modeling innate cellular phenotypes across a spectrum of patients exhibiting Parkinsonian features is essential.

Although the underlying cellular toxicities of most PD patients may converge on $\alpha$-syn, distinct mechanisms of disease progression would likely limit the applicability of any particular compound to certain patients. We have thus far focused on two main $\alpha$-syn lesions: the A53T mutation and the $\alpha$-syn gene triplication. Whether the phenotypes we observed, namely nitrosative stress or ERAD substrate accumulation, are going to be observed in diverse genetic backgrounds or sporadic cases remains to be seen. That said, the revolutionary advances in exome sequencing and genome editing, such as clustered regularly interspaced short palindromic repeats (CRISPR) technologies, will facilitate the analysis of otherwise genetically identical controls to make phenotypic characterization more reliable.

If our established innate phenotypes are not conserved across diverse genetic or sporadic patientderived cell lines, then we can return to yeast as an unbiased screening platform. For example, it is possible to generate $\alpha$-syn strains with additional genetic perturbations that may more accurately reflect the genetic predisposition of some PD patients. This approach could afford the opportunity to create simple "personalized" $\alpha$-syn toxicity yeast strains for additional chemical or genetic screening.

That said, Nedd4's apparent central position within the $\alpha$-syn network may increase its applicability to more diverse forms of PD. As mentioned earlier, other PD genetic risk factors function in endosomal trafficking, suggesting that this general pathway is critical to neuronal dysfunction and disease and may respond to a compound such as NAB2. In either case, establishing innate phenotypes in patient-derived neurons and modifying these phenotypes with small molecules will be critical to the success of disease-modifying therapies over those that manage downstream symptoms.

\section{Closing Remarks}

The rate of single nucleotide polymorphisms in the general population is much greater than previously thought. ${ }^{51}$ It is thus likely that most sporadic cases of PD actually derive from rare, low-penetrance mutations that are largely invisible to conventional genome-wide association studies. This, combined with enhancements in exome sequencing and a decrease in the cost of patient genome sequencing, will ultimately create an overwhelming wealth of information. Extracting meaningful information from these data will require diverse approaches and the ability to draw direct connections between nodes within complex cellular networks. Genetically tractable model organisms, such as yeast, nematode, and fruit flies, may be able to rapidly evaluate mechanisms of toxicity associated with perturbed, basic cellular pathways. Additionally, systems closer to human disease, such as patient-derived neurons, can help feed an iterative process of going between simple and complex model systems to elucidate mechanism, evaluate patient-specific drug targets, and test drug efficacy across patient-derived cell lines. As mentioned at the outset of this manuscript, we see value in bringing together diverse approaches to illuminate a black hole in drug discovery and public health.

Acknowledgments: We thank members of the Lindquist lab for comments on the manuscript and Tom DiCesare for help with illustrations.

\section{References}

1. Tardiff DF, Jui NT, Khurana V, et al. Yeast reveal a "druggable" Rsp5/Nedd4 network that ameliorates alpha-synuclein toxicity in neurons. Science 2013;342:979-983.

2. Chung CY, Khurana V, Auluck PK, et al. Identification and rescue of alpha-synuclein toxicity in Parkinson patient-derived neurons. Science 2013;342:983-987.

3. Khurana V, Lindquist S. Modelling neurodegeneration in Saccharomyces cerevisiae: why cook with baker's yeast? Nat Rev Neurosci 2010;11:436-449.

4. Outeiro TF, Lindquist S. Yeast cells provide insight into alphasynuclein biology and pathobiology. Science 2003;302:1772-1775.

5. Singleton AB, Farrer M, Johnson J, et al. Alpha-Synuclein locus triplication causes Parkinson's disease. Science 2003;302:841.

6. Chartier-Harlin MC, Kachergus J, Roumier C, et al. Alpha-synuclein locus duplication as a cause of familial Parkinson's disease. Lancet 2004;364:1167-1169.

7. Ibanez P, Bonnet AM, Debarges B, et al. Causal relation between alpha-synuclein gene duplication and familial Parkinson's disease. Lancet 2004;364:1169-1171. 
8. Pihlstrom L, Toft M. Genetic variability in SNCA and Parkinson's disease. Neurogenetics 2011;12:283-293.

9. Sotiriou S, Gibney G, Baxevanis AD, Nussbaum RL. A single nucleotide polymorphism in the 3'UTR of the SNCA gene encoding alpha-synuclein is a new potential susceptibility locus for Parkinson disease. Neurosci Lett 2009;461:196-201.

10. Cooper AA, Gitler AD, Cashikar A, et al. Alpha-synuclein blocks ER-Golgi traffic and Rab1 rescues neuron loss in Parkinson's models. Science 2006;313:324-328.

11. Yeger-Lotem E, Riva L, Su LJ, et al. Bridging high-throughput genetic and transcriptional data reveals cellular responses to alphasynuclein toxicity. Nat Genet 2009;41:316-323.

12. Gitler AD, Bevis BJ, Shorter J, et al. The Parkinson's disease protein alpha-synuclein disrupts cellular Rab homeostasis. Proc Natl Acad Sci U S A 2008;105:145-150.

13. Soper JH, Kehm V, Burd CG, Bankaitis VA, Lee VM. Aggregation of alpha-synuclein in S. cerevisiae is associated with defects in endosomal trafficking and phospholipid biosynthesis. J Mol Neurosci 2011;43:391-405.

14. Sancenon V, Lee SA, Patrick C, et al. Suppression of alphasynuclein toxicity and vesicle trafficking defects by phosphorylation at S129 in yeast depends on genetic context. Hum Mol Genet 2012;21:2432-2449.

15. Kisos H, Ben-Gedalya T, Sharon R. The clathrin-dependent localization of dopamine transporter to surface membranes is affected by alpha-synuclein. J Mol Neurosci. 2014;52:167-176.

16. Cheng F, Li X, Li Y, Wang C, Wang T, Liu G, et al. alpha-Synuclein promotes clathrin-mediated NMDA receptor endocytosis and attenuates NMDA-induced dopaminergic cell death. J Neurochem 2011;119:815-825.

17. Coune PG, Bensadoun JC, Aebischer P, Schneider BL. Rab1A over-expression prevents Golgi apparatus fragmentation and partially corrects motor deficits in an alpha-synuclein based rat model of Parkinson's disease. J Parkinsons Dis 2011;1:373-387.

18. Rendon WO, Martinez-Alonso E, Tomas M, Martinez-Martinez N, Martinez-Menarguez JA. Golgi fragmentation is Rab and SNARE dependent in cellular models of Parkinson's disease. Histochem Cell Biol 2013;139:671-684.

19. Thayanidhi N, Helm JR, Nycz DC, Bentley M, Liang Y, Hay JC. Alpha-synuclein delays endoplasmic reticulum (ER)-to-Golgi transport in mammalian cells by antagonizing ER/Golgi SNAREs. Mol Biol Cell 2010;21:1850-1863.

20. Su LJ, Auluck PK, Outeiro TF, et al. Compounds from an unbiased chemical screen reverse both ER-to-Golgi trafficking defects and mitochondrial dysfunction in Parkinson's disease models. Dis Model Mech 2010;3:194-208.

21. Oaks AW, Marsh-Armstrong N, Jones JM, Credle JJ, Sidhu A. Synucleins antagonize endoplasmic reticulum function to modulate dopamine transporter trafficking. PLoS One 2013;8: e70872.

22. Rockenstein E, Nuber S, Overk CR, et al. Accumulation of oligomer-prone alpha-synuclein exacerbates synaptic and neuronal degeneration in vivo. Brain 2014;137:1496-1513.

23. MacLeod DA, Rhinn $H$, Kuwahara $T$, et al. RAB7L1 interacts with LRRK2 to modify intraneuronal protein sorting and Parkinson's disease risk. Neuron 2013;77:425-439.

24. Paisan-Ruiz C, Jain S, Evans EW, et al. Cloning of the gene containing mutations that cause PARK8-linked Parkinson's disease. Neuron 2004;44:595-600.

25. Pankratz N, Wilk JB, Latourelle JC, et al. Genomewide association study for susceptibility genes contributing to familial Parkinson disease. Hum Genet 2009;124:593-605.

26. Vilarino-Guell C, Wider C, Ross OA, et al. VPS35 mutations in Parkinson disease. Am J Hum Genet. 2011;89:162-167.

27. Zimprich A, Benet-Pages A, Struhal W, et al. A mutation in VPS35, encoding a subunit of the retromer complex, causes lateonset Parkinson disease. Am J Hum Genet 2011;89:168-175.

28. Zimprich A, Biskup S, Leitner P, et al. Mutations in LRRK2 cause autosomal-dominant parkinsonism with pleomorphic pathology. Neuron 2004;44:601-607.
29. Vilarino-Guell C, Rajput A, Milnerwood AJ, et al. DNAJC13 mutations in Parkinson disease. Hum Mol Genet 2014;23:1794-1801.

30. Dumitriu A, Pacheco CD, Wilk JB, et al. Cyclin-G-associated kinase modifies alpha-synuclein expression levels and toxicity in Parkinson's disease: results from the GenePD Study. Hum Mol Genet 2011;20:1478-1487.

31. Gitler AD, Chesi A, Geddie ML, et al. Alpha-synuclein is part of a diverse and highly conserved interaction network that includes PARK9 and manganese toxicity. Nat Genet 2009;41:308-315.

32. Usenovic M, Tresse E, Mazzulli JR, Taylor JP, Krainc D. Deficiency of ATP13A2 leads to lysosomal dysfunction, alpha-synuclein accumulation, and neurotoxicity. J Neurosci. 2012;32:4240-4246.

33. Tsunemi T, Krainc D. Zn2+ dyshomeostasis caused by loss of ATP13A2/PARK9 leads to lysosomal dysfunction and alphasynuclein accumulation. Hum Mol Genet 2014;23:2791-2801.

34. Park JS, Koentjoro B, Veivers D, Mackay-Sim A, Sue CM. Parkinson's disease-associated human ATP13A2 (PARK9) deficiency causes zinc dyshomeostasis and mitochondrial dysfunction. Hum Mol Genet 2014;23:2802-2815.

35. Kong SM, Chan BK, Park JS, et al. Parkinson's disease-linked human PARK9/ATP13A2 maintains zinc homeostasis and promotes alpha-Synuclein externalization via exosomes. Hum Mol Genet 2014;23:2816-2833.

36. Cooper O, Seo H, Andrabi S, et al. Pharmacological rescue of mitochondrial deficits in iPSC-derived neural cells from patients with familial Parkinson's disease. Sci Transl Med 2012;4:141ra90.

37. Nguyen HN, Byers B, Cord B, et al. LRRK2 mutant iPSC-derived DA neurons demonstrate increased susceptibility to oxidative stress. Cell Stem Cell 2011;8:267-280.

38. Ryan SD, Dolatabadi N, Chan SF, et al. Isogenic human iPSC Parkinson's model shows nitrosative stress-induced dysfunction in MEF2-PGC1alpha transcription. Cell 2013;155:1351-1364.

39. Tardiff DF, Lindquist S. Phenotypic screens for compounds that target the cellular pathologies underlying Parkinson's disease. Drug Discov Today Technol 2013;10:e121-e128.

40. Swinney DC, Anthony J. How were new medicines discovered? Nat Rev Drug Discov 2011;10:507-519.

41. Smith AM, Ammar R, Nislow C, Giaever G. A survey of yeast genomic assays for drug and target discovery. Pharmacol Ther 2010;127:156-164.

42. Rotin D, Kumar S. Physiological functions of the HECT family of ubiquitin ligases. Nat Rev Mol Cell Biol 2009;10:398-409.

43. Takahashi K, Tanabe K, Ohnuki M, et al. Induction of pluripotent stem cells from adult human fibroblasts by defined factors. Cell 2007;131:861-872.

44. Sandoe J, Eggan K. Opportunities and challenges of pluripotent stem cell neurodegenerative disease models. Nat Neurosci 2013;16: 780-789.

45. Soldner F, Laganiere J, Cheng AW, et al. Generation of isogenic pluripotent stem cells differing exclusively at two early onset Parkinson point mutations. Cell 2011;146:318-331.

46. Kotzbauer PT, Giasson BI, Kravitz AV, et al. Fibrillization of alphasynuclein and tau in familial Parkinson's disease caused by the A53T alpha-synuclein mutation. Exp Neurol 2004;187:279-288.

47. Sidransky E. Gaucher disease and parkinsonism. Mol Genet Metab 2005;84:302-304.

48. Scott D, Roy S. alpha-Synuclein inhibits intersynaptic vesicle mobility and maintains recycling-pool homeostasis. J Neurosci. 2012;32:10129-10135.

49. Beilina A, Rudenko IN, Kaganovich A, et al. Unbiased screen for interactors of leucine-rich repeat kinase 2 supports a common pathway for sporadic and familial Parkinson disease. Proc Natl Acad Sci U S A 2014;111:2626-2631.

50. Tofaris GK, Kim HT, Hourez R, Jung JW, Kim KP, Goldberg AL. Ubiquitin ligase Nedd4 promotes alpha-synuclein degradation by the endosomal-lysosomal pathway. Proc Natl Acad Sci U S A 2011;108:17004-17009.

51. Casals F, Bertranpetit J. Genetics: human genetic variation, shared and private. Science 2012;337:39-40. 